\title{
Large Scale Fabrication of Periodical Bowl-like Micropatterns of Single Crystal ZnO
}

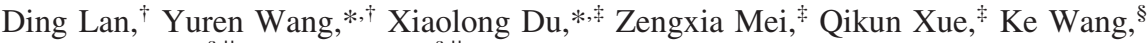 \\ Xiaodong Han, ${ }^{8, \|}$ and Ze Zhang ${ }^{8, \|}$ \\ National Microgravity Laboratory, Institute of Mechanics, Chinese Academy of Sciences, Beijing \\ 100190, China, Beijing National Laboratory for Condensed Matter Physics, Institute of Physics, \\ Chinese Academy of Sciences, Beijing 100190, China, Institute of Microstructure and Property of \\ Advanced Materials, Beijing University of Technology, Beijing 100022, China, and National Center of \\ Electron Microscope, Beijing, 100080, China
}

Received December 25, 2007; Revised Manuscript Received May 21, 2008

\begin{abstract}
Nanostructured $\mathrm{ZnO}$ materials are of great significance for their potential applications in photoelectronic devices, light-emitting displays, catalysis and gas sensors. In this paper, we report a new method to produce large area periodical bowl-like micropatterns of single crystal $\mathrm{ZnO}$ through aqueous-phase epitaxial growth on a $\mathrm{ZnO}$ single crystal substrate. A self-assembled monolayer of polystyrene microspheres was used as a template to confine the epitaxial growth of single crystal $\mathrm{ZnO}$ from the substrate, while the growth morphology was well controlled by citrate anions. Moreover, it was found that the self-assembled monolayer of colloidal spheres plays an important role in reduction of the defect density in the epitaxial $\mathrm{ZnO}$ layer. Though the mechanism is still open for further investigation, the present result indicates a new route to suppress the dislocations in the fabrication of single crystal $\mathrm{ZnO}$ film. A predicable application of this new method is for the fabrication of two-dimensional photonic crystal structures on light emitting diode surfaces.
\end{abstract}

Nanostructured $\mathrm{ZnO}$ materials have been extensively studied due to their potential applications in photoelectronic devices, light-emitting displays, catalysis and gas sensors. Many kinds of $\mathrm{ZnO}$ nanostructures, such as nanoflowers, ${ }^{1}$ nanorods ${ }^{2,3}$ and nanotetropods, ${ }^{4,5}$ are reported in the literature. More complex nanostructured $\mathrm{ZnO}$ films were fabricated by utilizing a sequential nucleation and growth method. ${ }^{6} \mathrm{~A}$ new trend is to integrate individual nanostructured $\mathrm{ZnO}$ blocks into a large highly ordered assembly which is crucial for achieving adequate device performance. Hsu et al. $^{7}$ reported the fabrication of hierarchical micropatterns by the selective growth of $\mathrm{ZnO}$ nanocrystals. By utilizing a self-assembled microsphere monolayer as a template, periodic $\mathrm{ZnO}$ nanorod arrays ${ }^{8}$ were successfully obtained. Bowl-like microstructured $\mathrm{ZnO}$ and $\mathrm{TiO}_{2}$ were also reported in the literature, ${ }^{9-11}$ in which the methods of nanoparticles/colloidal spheres codeposition, electrochemical deposition and atomic layer deposition were used to construct the bowl-like porous skeletons. In order to get periodic structures, controlling nucleation sites and growth morphology are important. Several methods ${ }^{12-14}$ have been put forward to produce large area $\mathrm{ZnO}$ nanorod arrays through fluid flow driven self-assembly or growth on patterned $\mathrm{Si}$ substrate. A few researchers ${ }^{15,16}$ reported the synthesis of single-crystalline patterned $\mathrm{ZnO}$ structure through solution-based epitaxial growth. In this paper, we report a new method to produce large area periodical bowl-like micropatterns (PBLM) of single crystal $\mathrm{ZnO}$ through aqueous-phase epitaxial growth on a $\mathrm{ZnO}$ single crystal substrate. A self-assembled monolayer of polystyrene (PS) microspheres was used as a template to confine the epitaxial growth of single crystal $\mathrm{ZnO}$ from the substrate and form

* Correspondence should be addressed to (Y.W.) E-mail: wangyr@imech.ac.cn. Phone: +086-010-82544091. Fax: +086-010-82544096. (X.D.) E-mail: xldu@ aphy.iphy.ac.cn. Phone: +086-010-82649035. Fax: +086-010-82649228.

Institute of Mechanics, Chinese Academy of Sciences.

Beijing National Laboratory for Condensed Matter Physics, Institute of Physics, Chinese Academy of Sciences.

$\$$ Beijing University of Technology.

"National Center of Electron Microscope.
PBLM. Citrate anions were employed to control the growth morphology which was proven to be crucial to obtain the presented micropattern. On the basis of the knowledge of the authors, this is the first time for a report on the synthesis of such a micropattern of single crystal $\mathrm{ZnO}$.

It is well-known that the light-emitting efficiency of a lightemitting diode (LED) is greatly limited by the internal reflection of the emitting light at the solid/air interface. It has been demonstrated that constructing photonic crystal (PC) structures ${ }^{17-23}$ on the LED surface is a promising way to solve the problem. The present study provided a simple route to construct twodimensional PC structure on the surface of single crystal $\mathrm{ZnO}$ substrate. Moreover, our method is based on an aqueous-phase synthesis of nanostructured $\mathrm{ZnO}$ and a totally chemical procedure for the preparation of patterned substrate. It offers the potential for low-cost, industrial-scale manufacturing ${ }^{6}$ of PCassistant LED devices. In addition, it was surprisingly revealed that the PS monolayer, which was used as a template for subsequent epitaxial growth, can obviously reduce the defect density and obtain high-quality epitaxial film. Different from the extensively reported "window" effect and lateral epitaxial overgrowth (LEO) technique by which threading dislocations could be suppressed, ${ }^{15,16}$ the confinement effect of adjacent PS spheres may provide a new mechanism for efficiently reducing defect density in the epitaxial $\mathrm{ZnO}$ thin film.

Figure 1 shows a schematic of our experimental procedure. The single crystalline $\mathrm{ZnO}(0001)$ films with $\mathrm{Zn}$-polarity were grown on $\mathrm{Si}(111)$ wafers by molecular beam epitaxy (MBE) ${ }^{24}$ and were used as seeds in the subsequent hydrothermal growth of micropatterned $\mathrm{ZnO}$ film. The thickness of the MBE-grown $\mathrm{ZnO}$ film was $900 \mathrm{~nm}$. PSs (10\% in water) with a diameter of $1 \mu \mathrm{m}$ and $583 \mathrm{~nm}$ were self-assembled to make a monolayer mask $^{25}$ on the MBE-grown $\mathrm{ZnO}(0001) / \mathrm{Si}(111)$ template. Then the substrate was heated at $90{ }^{\circ} \mathrm{C}$ for $15 \mathrm{~min}$ to sinter the colloidal spheres before the hydrothermal growth. The prepared monolayers of PSs were used as template film, which is shown in Figure 1I. The specimen was mounted upside-down in a 


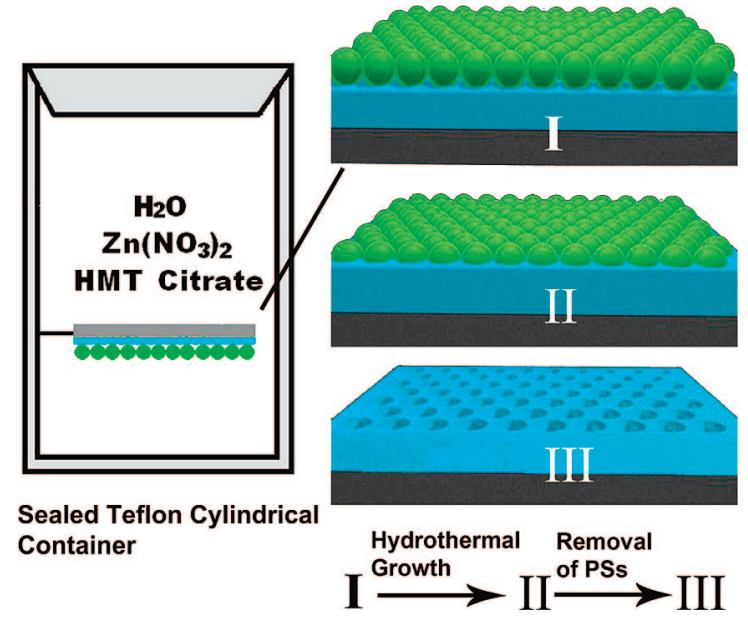

Figure 1. A schematic of the experimental procedure. The left panel shows the reaction container, the components of the precursor, and the status of the substrate; the brief fabrication procedure of ordered porous single crystal $\mathrm{ZnO}$ is shown in the right panel.

sealed Teflon cylindrical container, containing a 30-mL solution of $0.3 \mathrm{M}$ methenamine $\left(\mathrm{C}_{6} \mathrm{H}_{12} \mathrm{~N}_{4}, \mathrm{HMT}\right), 0.03 \mathrm{M}$ zinc nitrate hexahydrate $\left(\mathrm{Zn}\left(\mathrm{NO}_{3}\right)_{2} \cdot 6 \mathrm{H}_{2} \mathrm{O}\right)$ and $1.5 \mathrm{mg}$ of sodium citrate. The reaction temperature and time are $60{ }^{\circ} \mathrm{C}$ and $36 \mathrm{~h}$, respectively. The chemical reaction procedure was described in the literature. ${ }^{26}$ The growth thickness can be controlled by the hydrothermal growth time. $\mathrm{ZnO}$ growth in the hydrothermal crucible was carefully controlled so as to enhance permeation of precursor solution and nucleation of $\mathrm{ZnO}$ in the interstitial space among the PSs of template film. Therefore, the PSs were buried in the as-grown single-crystal $\mathrm{ZnO}$, which is shown schematically in Figure 1II. After removal of the PSs (burned off at $450^{\circ}$ or dissolved in toluene), the periodical bowl-like single-crystal $\mathrm{ZnO}$ film could be obtained, which is shown schematically in Figure 1III.

Figure $2 \mathrm{a}-\mathrm{c}$ shows the SEM images of the synthesized $\mathrm{ZnO}$ film of PBLM. The hydrothermal growth was carried out for $36 \mathrm{~h}$ at the growth temperature of $60{ }^{\circ} \mathrm{C}$. When the growth process was finished, the PSs were burnt off and left bowl-like air pores in the grown film. Figure 2 a clearly shows that the air pores are arranged in a close-packed hexagonal-pattern, while the surface of the PBLM film was kept flat and smooth. To reveal the fine structure of PBLM film, the magnification SEM image is shown in Figure 2b. Due to the close-packing of the PSs, the air pores are tightly connected to each other through the small tunnels between two adjacent air pores. The crosssectional SEM image of the sample is shown in Figure 2c. The image shows that the top porous layer integrated into the seed substrate very well. Almost no interface between the porous layer and the seed substrate could be distinguished on the image. It indicated that the epitaxial growth of single crystal $\mathrm{ZnO}$ was achieved. The diameter of the air pores is $1030 \mathrm{~nm}$, which is consistent with the size of the PSs. This implies that no obvious shrinkage occurred in the synthesis of the porous film.

In the above procedure, a key issue is how to keep growth of $\mathrm{ZnO}$ homogeneous and smooth in the interstitial space among PSs during the growing process. It is well-known that $\mathrm{ZnO}$ grows rapidly in the [0001] direction. The growth rate of $\mathrm{ZnO}$ crystal should be well controlled in the experiment. The interstitial space among PSs can be divided into two parts, as shown in Figure 3a. It should be noted that the interstitial space is only several nanometers. Part I region is exposed to the solution directly. $\mathrm{Zn}^{2+}$ and HMT in the solution can readily
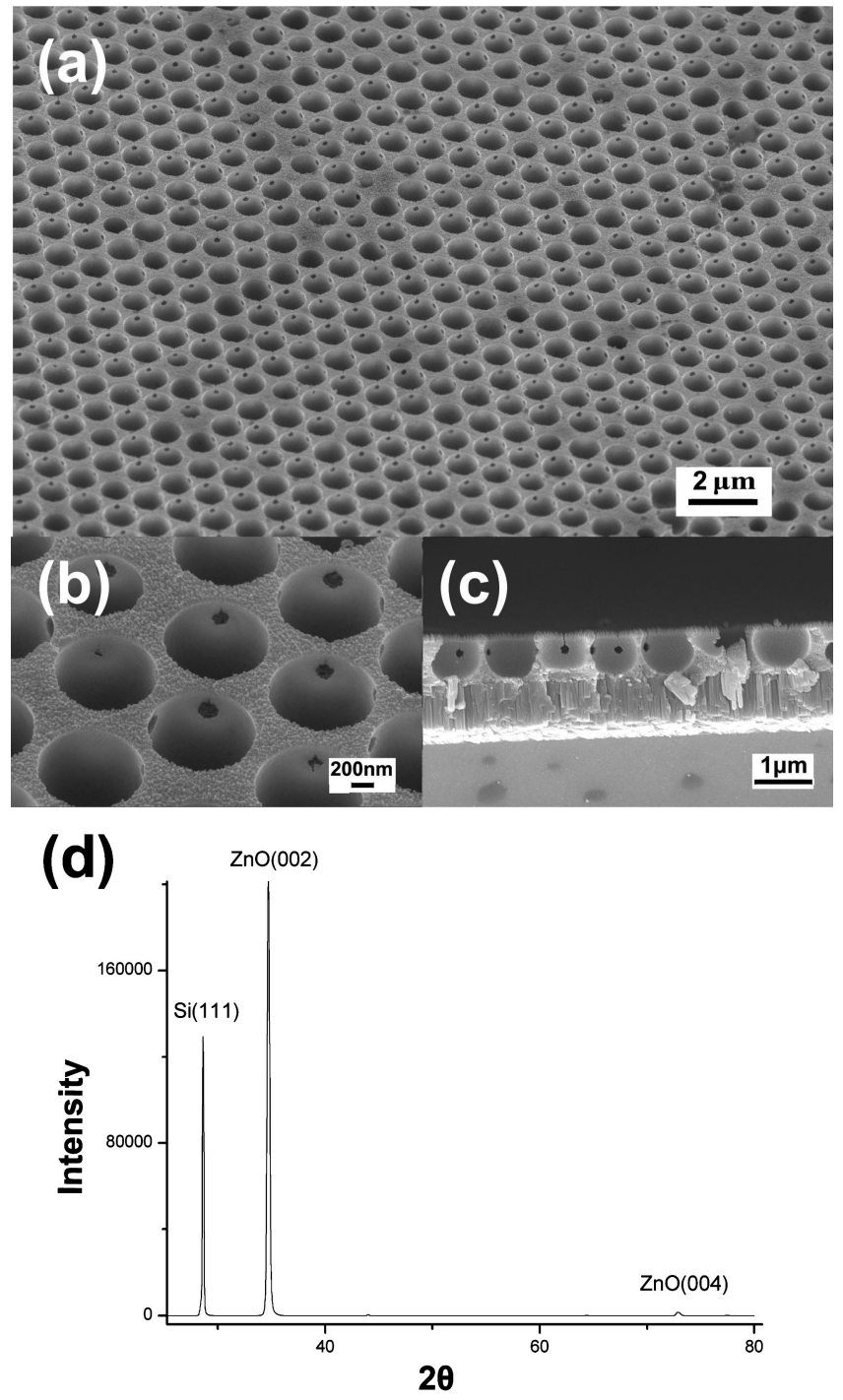

Figure 2. SEM images of a periodical bowl-like micropattern on single crystal $\mathrm{ZnO}$ substrate with PSs of $1 \mu \mathrm{m}$ as the template. (a) The surface morphology with the citrate as the growth rate buffer agent. The hydrothermal growth time and the temperature are $36 \mathrm{~h}$ and $60{ }^{\circ} \mathrm{C}$, respectively. (b) Magnification SEM image of (a), (c) cross-sectional SEM image of (a), (d) X-ray diffraction spectrum of (a).

diffuse to this region. On the contrary, part II region is shadowed by the PSs. Therefore, it is difficult for the solute to enter this region. Figure $3 \mathrm{~b}$ shows the projection areas of part I and part II on the seed substrate. It is easy to understand that the easy diffusion of solute into region I induced the leading growth on

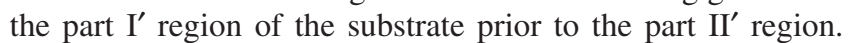
Figure 3c,d shows the SEM images of the sample growing in the precursor solution without adding sodium citrate. The surface morphology of the sample is ragged. This implied that the growth rate in region $\mathrm{I}^{\prime}$ is much faster than that in region $\mathrm{II}^{\prime}$. Once the crystal growth in region $\mathrm{I}^{\prime}$ approaches the pore throat, it will further hinder the solute diffusion into the niche surrounded by three close-packed PS spheres. The crystal growth in region II' was seriously suppressed. In order to solve the problem, it is necessary to suppress the growth in the $\mathrm{ZnO}$ [0001] direction so that the precursor solution can diffuse into the part II region. It has been demonstrated that citrate anions can control the crystal morphologies by adsorbing strongly on mineral surfaces ${ }^{27}$ and altering the mineral growth behavior. ${ }^{28}$ The citrate ions are absorbed preferentially on the (0001) surface 


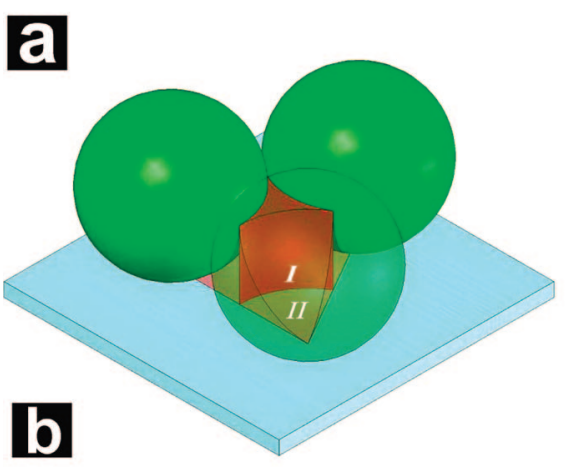

\section{C}
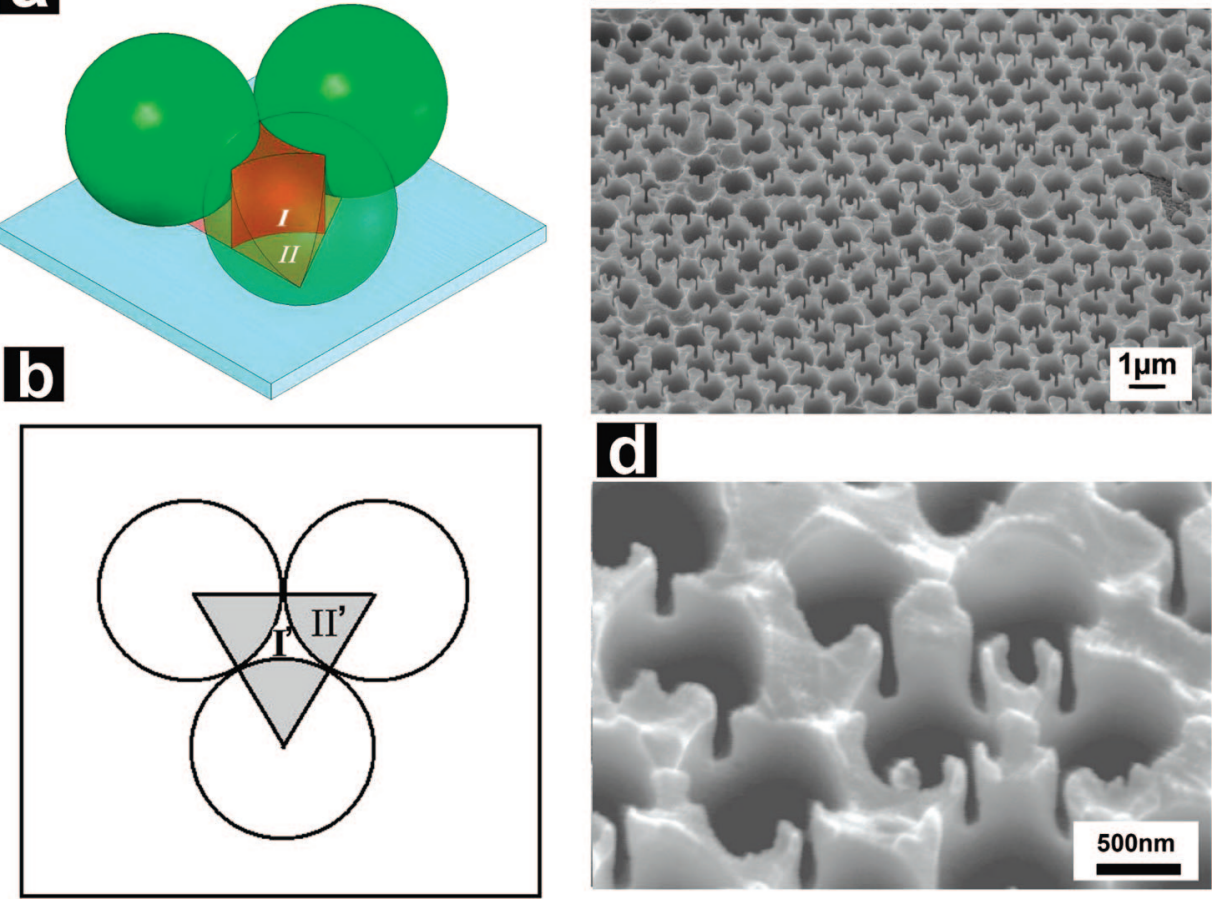

Figure 3. The nanospace and its effect on the epitaxial growth of $\mathrm{ZnO}$. (a) The nanospace confined by substrate and PSs. (b) Two regions of the seed substrate for $\mathrm{ZnO}$ growth, (c) the sample that grew hydrothermally in the precursor solution without sodium citrate, (d) magnification of (c).

of $\mathrm{ZnO}$ and thus inhibit the crystal growth along the [0001] orientations. ${ }^{29}$ In the experiment, we added a suitable amount of citrate in the solution to slow down the crystal growth along the [0001] orientation. The quality of the single-crystal porous $\mathrm{ZnO}$ film is improved remarkably (as shown in Figure 2a). Adding citrate ions into the precursor solution made the crystal growth in the pore niche more homogeneous.

We studied the crystal structure of the synthesized porous $\mathrm{ZnO}$ film by X-ray diffraction spectra (XRD), which is shown in Figure 2d. It can be seen that only diffraction peaks belonging to $\mathrm{ZnO}$ [001] reflections appeared on the XRD spectra, which is very consistent with the normal XRD spectra from single crystal $\mathrm{ZnO}$ film. It demonstrated that the porous $\mathrm{ZnO}$ film preserved the single crystal structure of the seed substrate and strongly orientated in the [0001] direction, even if the PSs template was used in the hydrothermal growth.

TEM and high-resolution electron microscopy (HREM) experiments were used to characterize the microstructural features of the two epitaxial $\mathrm{ZnO}$ layers, parent epitaxial layer (P) and the daughter epitaxial layer (D), at the atomic scale. Figure $4 \mathrm{a}$ is a low magnification image of the $\mathrm{ZnO}$ thin film covered with a monolayer of PSs. The parent $\mathrm{ZnO}$ layer is indicated by 1 and the daughter epitaxial $\mathrm{ZnO}$ thin film is indicated by 2 . An obvious interface between zone 1 and zone 2 was observed as indicated by the white dash line in Figure $4 \mathrm{a}$. The selected area diffraction patterns taken from areas $\mathrm{B}, \mathrm{C}$ and $\mathrm{D}$ are shown as b, c and d, respectively. It reveals that the parent epitaxial layer is single crystalline $\mathrm{ZnO}$ on $\mathrm{Si}$ substrate. The epitaxial growth is with an orientation relationship of $(0001)_{\mathrm{ZnO}} \|(\overline{1} 1 \overline{1})_{\mathrm{Si}}$ and $[2 \overline{1} \overline{1} 0]_{\mathrm{ZnO}}$ II $[011]_{\mathrm{Si}}$. The HREM image shown in Figure 4e (taken from the framed region E of Figure 4a) reveals that the features of parent epitaxial layer is high quality single crystalline $\mathrm{ZnO}$ film. The parent $\mathrm{ZnO}$ film shows excellent epitaxial characters at the atomic scale. The white dots can be regarded as atomic chain projects according to the electron diffraction theory ${ }^{30}$ with a first-order approximation. The simulated HREM image and the corresponding experimentally recorded HREM image are shown as insets in Figure 4e and they agree with each other very well. The parent epitaxial layer was through two transition layers as indicated as $\mathrm{SiO} x$ which is typical in conventional MBE growth of oxides on Si substrate. ${ }^{31}$ It is noteworthy to pay attention to the "column-like" contrast in the parent epitaxial $\mathrm{ZnO}$ thin film layer. This column-like contrast terminates at the interface with the daughter $\mathrm{ZnO}$ epitaxial layer. The column-like contrast is suggested to derive from elastic field strains. No high or low angle grain boundaries were revealed in these column-like $\mathrm{ZnO}$ thin films. Figure $4 \mathrm{f}-\mathrm{h}$ shows the HREM images of the 2D macroporous daughter epitaxial $\mathrm{ZnO}$ thin film which were hydrothermally grown on the MBE-grown parent $\mathrm{ZnO}$ template. The fast Fourier transformation (FFT) diffraction patterns are shown in the corresponding figures. The enlarged HREM images (Figure $4 \mathrm{f}-\mathrm{h}$ ) show the structural details of the framed areas F, G, H respectively in Figure $4 \mathrm{a}$ at the atomic level. Area $\mathrm{H}$ in Figure $4 \mathrm{a}$ contains the parent-daughter $\mathrm{ZnO}$ interface zone and shows the nucleation and transition characters of the daughter $\mathrm{ZnO}$ thin film on the parent. Figure $4 \mathrm{~h}$ shows the microstructural characters of the transition zone which indicates heavy lattice distortions. The interface transition zone is about $50-100 \mathrm{~nm}$ thick. Through this interface transition zone, the strain was released quickly and the upgrown $\mathrm{ZnO}$ thin films show high quality single crystalline features. Across the parent-daughter interface, the columnlike contrast in the parent zone disappeared sharply. As revealed in Figure $4 \mathrm{f}$ (the side of the PS) and Figure $4 \mathrm{~g}$ (the bottom of the PS), both PS/ZnO interface regions (bottom and side) show very high quality single crystalline $\mathrm{ZnO}$ characters. The above results suggest that the PS monolayer suppressed the formation of threading defects or released the 


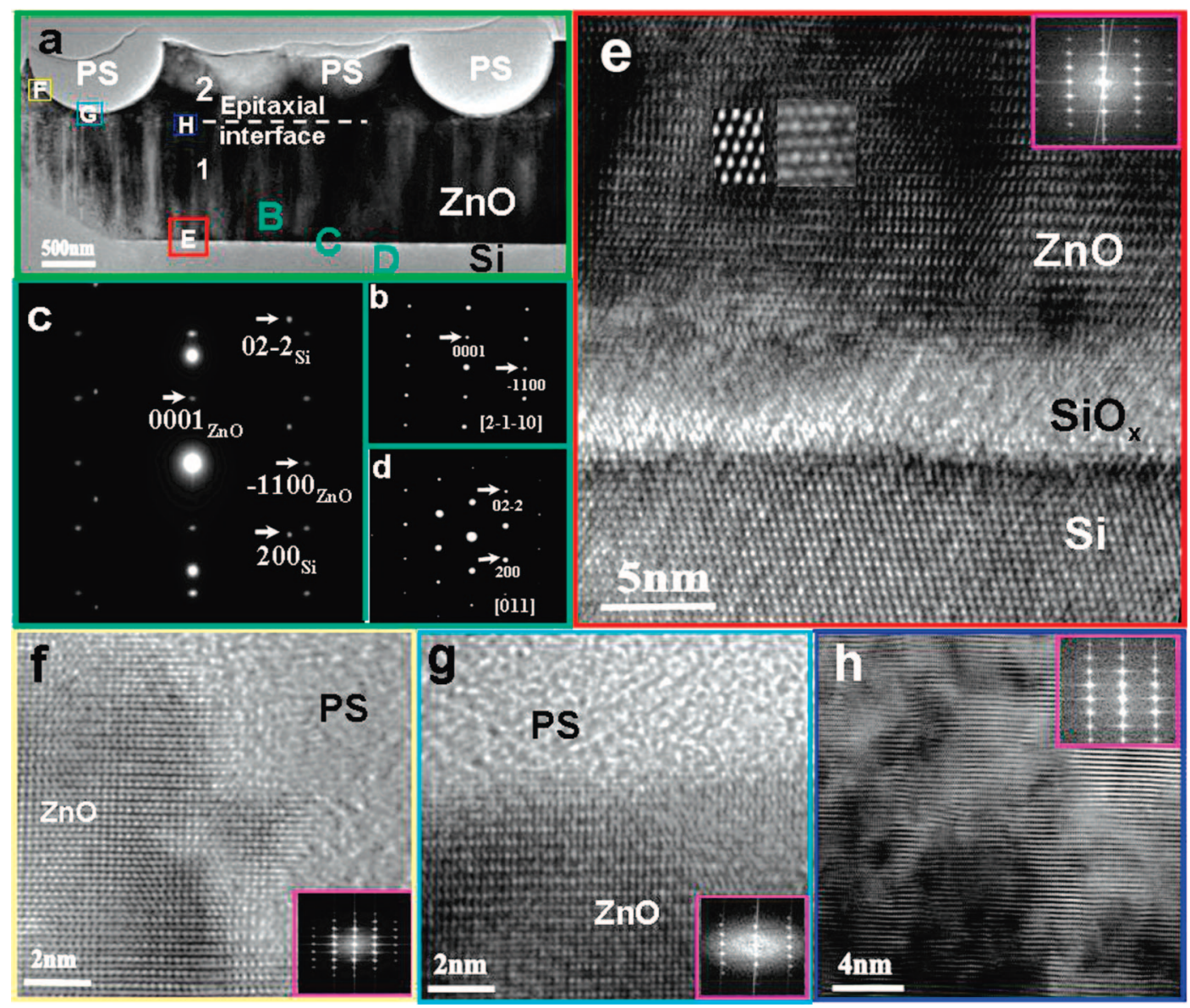

Figure 4. (a) TEM image shows the interface region between the epitaxial $\mathrm{ZnO}$ layer of the bowl-like micropattern and the seed single crystal $\mathrm{ZnO}$ film grown on a silicon (111) substrate. The selected area diffraction patterns corresponding to B, C, and D marked regions in image (a) were shown in panels (b), (c) and (d), separately. High-resolution electron microscopy (HREM) images corresponding to E, F, G and H marked regions in image (a) were shown in panels (e), (f), (g) and (h), respectively.

threading defects along the PS interface in the hydrothermally grown $\mathrm{ZnO}$ film. Interestingly, almost perfect $\mathrm{ZnO} / \mathrm{PS}$ interface without any detectable line defects at the atomic scale indicates the extreme benign interface structure of the PS for $\mathrm{ZnO}$ ordering growth. These results suggested the threading dislocations in LEO mode are bent to and/or confined in the masked region due to the change of growth direction from vertical to lateral. ${ }^{32}$ Therefore, window region itself in the LEO mode just provided a passage for the subsequent epitaxial growth and has no effect on the defect reduction. In fact, defects directly go through the window region and extend to the up-grown epitaxial region in $\mathrm{ZnO}$ LEO ${ }^{15}$ while much less threading defects were observed in the overgrown wing area. For this reason, the second growth and interlaced windows were used in $\mathrm{ZnO}$ LEO to guarantee a homogeneous low-defect growth. It seems that the colloidal sphere monolayer used in our experiment can be regarded as periodical mask in LEO. However, it is noteworthy that the high quality single crystal porous film was grown in the interstitial sites among the close packed colloidal spheres, other than overgrowth from the colloidal sphere monolayer. Therefore, the lateral growth mechanism cannot be used to explain the defect reduction observed in our experiment. The interstitial site surrounded by three close packed colloidal spheres has a complicated geometrical shape, as shown schematically in Figure 3a. It has nanometer-sized dimension which strongly limited solvent diffusion and selective nucleation in the epitaxial growth. It is suggested that the defect reduction mechanism has a strong relationship to the nanospace-confined diffusion process and the selective nucleation procedure.

In summary, we developed a new method to produce the large area periodical bowl-like micropattern (PBLM) of single crystal $\mathrm{ZnO}$ through an aqueous-phase epitaxial growth on a $\mathrm{ZnO}$ single crystal substrate. Self-assembled monolayer of polystyrene microspheres was used as a template to confine the epitaxial growth of single crystal $\mathrm{ZnO}$ from the substrate, while the growth morphology was well controlled by citrate anions. Moreover, the self-assembled monolayer of colloidal spheres plays an important role in reduction of the defect density in the epitaxial $\mathrm{ZnO}$ layer. Though the mechanism is still open for further investigation, the present result indicates a new route to suppress the dislocations in the fabrication of single crystal $\mathrm{ZnO}$ film. A predicable application of this new method is the fabrication of 2D photonic crystal structures on LED surfaces.

Acknowledgment. This project is supported by the Knowledge Innovation Program of the Chinese Academy of Sciences (Grant No KJCX2-SW-L05) and The National Science Foundation of China (Grant No 50532090).

Supporting Information Available: Fabrication of single crystal $\mathrm{ZnO}$ substrate, overgrowth of the periodical bowl-like single-crystal $\mathrm{ZnO}$, the periodical bowl-like single-crystal $\mathrm{ZnO}$ of $583 \mathrm{~nm}$ and characterization of the experiments. This material is available free of charge via the Internet at http://pubs.acs.org. 


\section{References}

(1) Wang, Z.; Qian, X. F.; Yin, J.; Zhu, Z. K. Large-scale fabrication of tower-like, flower-like, and tube-like $\mathrm{ZnO}$ arrays by a simple chemical solution route. Langmuir 2004, 20 (8), 3441-3448.

(2) Choy, J. H.; Jang, E. S.; Won, J. H.; Chung, J. H.; Jang, D. J.; Kim, $\mathrm{Y}$. W. Soft solution route to directionally grown $\mathrm{ZnO}$ nanorod arrays on Si wafer; room-temperature ultraviolet laser. Adv. Mater. 2003, 15 (22), 1911-1914.

(3) Park, W. I.; Yi, G. C.; Kim, J. W.; Park, S. M. Schottky nanocontacts on $\mathrm{ZnO}$ nanorod arrays. Appl. Phys. Lett. 2003, 82 (24), 4358-4360.

(4) Newton, M. C.; Warburton, P. A. ZnO tetrapod nanocrystals. Mater. Today 2007, 10 (5), 50-54.

(5) Chen, Z.; Shan, Z. W.; Cao, M. S.; Lu, L.; Mao, S. X. Zinc oxide nanotetrapods. Nanotechnology 2004, 15 (3), 365-369.

(6) Sounart, T. L.; Liu, J.; Voigt, J. A.; Hsu, J. W.; Spoerke, E. D.; Tian, Z.; Jiang, Y. B. Sequential nucleation and growth of complex nanostructured films. Adv. Funct. Mater. 2006, 16 (3), 335-344.

(7) Hsu, J. W.; Tian, Z. R.; Simmons, N. C.; Matzke, C. M.; Voigt, J. A.; Liu, J. Directed spatial organization of zinc oxide nanorods. Nano Lett. 2005, 5 (1), 83-86.

(8) Liu, D. F.; Xiang, Y. J.; Wu, X. C.; Zhang, Z. X.; Liu, L. F.; Song, L.; Zhao, X. W.; Luo, S. D.; Ma, W. J.; Shen, J.; Zhou, W. Y.; Wang, G.; Wang, C. Y.; Xie, S. S. Periodic ZnO nanorod arrays defined by polystyrene microsphere self-assembled monolayers. Nano Lett. 2006, 6 (10), 2375-2378.

(9) Cao, B. Q.; Cai, W. P.; Sun, F.Q.,.; Li, Y.; Lei, Y.; Zhang, L. D. Fabrication of large-scale zinc oxide ordered pore arrays with controllable morphology. Chem. Commun. 2004, 14, 1604-1605.

(10) Liu, Z. F.; Jin, Z. G.; Li, W.; Qiu, H. J. Assembly of ordered ZnO porous thin films by cooperative assembly method using polystyrene spheres and Ultrafine $\mathrm{ZnO}$ particles. Mater. Res. Bull. 2006, 41 (1), $119-127$

(11) Xu, D. W.; Graugnard, E.; King, J. S.; Zhong, L. W.; Summers, C. J. Large-scale fabrication of ordered nanobowl arrays. Nano Lett. 2004, 4 (11), 2223-2226

(12) Sun, B. Q.; Sirringhaus, H. Surface tension and fluid flow driven selfassembly of ordered $\mathrm{ZnO}$ nanorod films for high-performance field effect transistors. J. Am. Chem. Soc. 2006, 128 (50), 16231-16237.

(13) Kim, Y. J.; Lee, C. H.; Hong, Y. J.; Yi, G. C.; Kim, S. S.; Cheong, $\mathrm{H}$. Controlled selective growth of $\mathrm{ZnO}$ nanorod and microrod arrays on Si substrates by a wet chemical method. Appl. Phys. Lett. 2006, 89, (16312816)

(14) Tak, Y.; Yong, K. J. Controlled growth of well-aligned $\mathrm{ZnO}$ nanorod array using a novel solution method. J. Phys. Chem. B 2005, 109 (41), 19263-19269.

(15) Andeen, D.; Kim, J. H.; Lange, F. F.; Goh, G. K. L.; Tripathy, S. Lateral epitaxial overgrowth of $\mathrm{ZnO}$ in water at 90 degrees C. Adv. Funct. Mater. 2006, 16, 799-804.

(16) Kim, J. H.; Andeen, D.; Lange, F. F. Hydrothermal growth of periodic, single-crystal $\mathrm{ZnO}$ microrods and microtunnels. Adv. Mater. 2006, $18,2453$.

(17) Byeon, K. J.; Hwang, S. Y.; Lee, H. Fabrication of two-dimensional photonic crystal patterns on GaN-based light-emitting diodes using thermally curable monomer-based nanoimprint lithography. Appl. Phys. Lett. 2007, 91, (0911069)

(18) Kim, S. H.; Lee, K. D.; Kim, J. Y.; Kwon, M. K.; Park, S. J. Fabrication of photonic crystal structures on light emitting diodes by nanoimprint lithography. Nanotechnology 2007, 18, (0553065)

(19) Cho, H. K.; Jang, J.; Choi, J. H.; Choi, J.; Kim, J.; Lee, J. S.; Lee, B.; Choe, Y. H.; Lee, K. D.; Kim, S. H.; Lee, K.; Kim, S. K.; Lee, Y. H. Light extraction enhancement from nano-imprinted photonic crystal GaN-based blue light-emitting diodes. Opt. Express 2006, 14 (19), $8654-8660$

(20) Lin, C. H.; Tsai, J. Y.; Kao, C. C.; Kuo, H. C.; Yu, C. C.; Lo, J. R.; Leung, K. M. Enhanced light output in InGaN-based light-emitting diodes with onmidirectional one-dimensional photonic crystals. Jpn. J. Appl. Phys. Part 1 2006, 45 (3A), 1591-1593.

(21) Kim, D. H.; Cho, C. O.; Roh, Y. G.; Jeon, H.; Park, Y. S.; Cho, J.; Im, J. S.; Sone, C.; Park, Y.; Choi, W. J.; Park, Q. H. Enhanced light extraction from GaN-based light-emitting diodes with holographically generated two-dimensional photonic crystal patterns. Appl. Phys. Lett. 2005, 87, (20350820)

(22) Wierer, J. J.; Krames, M. R.; Epler, J. E.; Gardner, N. F.; Craford, M. G.; Wendt, J. R.; Simmons, J. A.; Sigalas, M. M. InGaN/GaN quantum-well heterostructure light-emitting diodes employing photonic crystal structures. Appl. Phys. Lett. 2004, 84 (19), 3885-3887.

(23) Oder, T. N.; Kim, K. H.; Lin, J. Y.; Jiang, H. X. III-nitride blue and ultraviolet photonic crystal light emitting diodes. Appl. Phys. Lett. 2004, 84 (4), 466-468.

(24) Wang, X. N.; Wang, Y.; Mei, Z. X.; Dong, J.; Zeng, Z. Q.; Yuan, H. T.; Zhang, T. C.; Du, X. L.; Jia, J. F.; Xue, Q. K.; Zhang, X. N.; Zhang, Z.; Li, Z. F.; Lu, W. Low-temperature engineering for highquality epitaxy of $\mathrm{ZnO}$ film on $\mathrm{Si}(111)$ substrate. Appl. Phys. Lett. 2007, 90, 151912.

(25) Wang, X. D.; Summers, C. J.; Wang, Z. L. Large-scale hexagonalpatterned growth of aligned $\mathrm{ZnO}$ nanorods for nano-optoelectronics and nanosensor arrays. Nano Lett. 2004, 4, 423-426.

(26) Vayssieres, L. Growth of arrayed nanorods and nanowires of $\mathrm{ZnO}$ from aqueous solutions. Adv. Mater. 2003, 15, 464-466.

(27) Lopez-Macipe, A.; Gomez-Morales, J.; Rodriguez-Clemente, R. The role of $\mathrm{pH}$ in the adsorption of citrate ions on hydroxyapatite. J. Colloid Interface Sci. 1998, 200, 114-120.

(28) Liu, C.; Huang, P. M. Atomic force microscopy and surface characteristics of iron oxides formed in citrate solutions. Soil Sci. Soc. Am. J. 1999, 63, 65-72.

(29) Tian, Z. R. R. Complex and oriented $\mathrm{ZnO}$ nanostructures. Nat. Mater. 2003, 2, 821-826.

(30) Cowley J. M. Diffraction Physics; North-Holland Publishing Company: New York, 1975.

(31) Hu, X. M.; Li, H.; Liang, Y.; Wei, Y.; Yu, Z.; Marshall, D.; Edwards, J.; Droopad, R.; Zhang, X.; Demkov, A. A.; Moore, K.; Kulik, J. The interface of epitaxial SrTiO3 on silicon: in situ and ex situ studies. Appl. Phys. Lett. 2003, 82, 203-205.

(32) Tanaka, S.; Honda, Y.; Sawaki, N.; Hibino, M. Structural characterization of GaN laterally overgrown on a (111) Si substrate. Appl. Phys. Lett. 2001, 79, 955-957.

\section{CG7012683}

